\title{
Tres nuevas especies y nuevos registros de Ladoffa de Panamá (Insecta: Hemiptera: Cicadellidae: Cicadellinae)
}

\section{Three new species and new records of Ladoffa from Panama (Insecta: Hemiptera: Cicadellidae: Cicadellinae)}

\author{
Pedro W. Lozada ${ }^{1}$ y Paul H. Freytag ${ }^{2}$
}

\begin{abstract}
1 Laboratorio de Entomología, Centro de Diagnóstico de Sanidad Centro de Dignostico de Sanidad Cegetal, SENASA, Av. La Molina 1915, Lima 12, y Departamento de Entomología, Museo de Historia Natural, Universidad Nacional Mayor de San Marcos, Av. Arenales 1256, Apartado 14-0434, Lima 14, PERU. Email Pedro Lozada: plozada21@gmail.com

2 Department of Entomology, University of Kentucky, Lexington, KY 40546-0091, U.S.A.
\end{abstract}

Presentado: 30/04/2010 Aceptado: $\quad 13 / 10 / 2010$ Publicado online: $14 / 12 / 2010$

\begin{abstract}
Resumen
Se describen e ilustran tres nuevas especies panameñas del género Ladoffa: woldai Lozada \& Freytag sp.nov., lamasi Lozada \& Freytag sp.nov. y grandis Lozada \& Freytag sp.nov. Asimismo, se dan a conocer nuevos registros para Panamá de las especies variolaria Young, donsana Young, rubriguttata (Walker), elauta Young, dependens Young, uncata Young y trifasciata Cavichioli \& Chiamolera.
\end{abstract}

Palabras clave: Hemiptera, Cicadellidae, Cicadellinae, Ladoffa, nuevas especies, nuevos registros, Panamá.

\section{Abstract}

Three new Panamanian species of the genus Ladoffa: woldai Lozada \& Freytag sp.nov., lamasi Lozada \& Freytag sp.nov. and grandis Lozada \& Freytag sp.nov., are described and illustrated herein. Likewise, new Panamanian records for the species variolaria Young, donsana Young, rubriguttata (Walker), elauta Young, dependens Young, uncata Young and trifasciata Cavichioli \& Chiamolera, are reported.

Keywords: Hemiptera, Cicadellidae, Cicadellinae, Ladoffa, new species, new records, Panama.

\section{Introducción}

El género Ladoffa fue creado por Young (1977) con 24 especies conocidas de Centro y Sudamérica. Lozada (1993) describió una nueva especie de Perú, Ladoffa aguilari Lozada, encontrada posteriormente en Guyana (Basset \& Charles 2000). Cavichioli \& Chiamolera (2001) describieron tres nuevas especies más de Brasil, Guyana y Panamá. Su distribución varía desde México hasta Bolivia, desde bosques montanos a bajos.

En este artículo, se describen 3 nuevas especies de Ladoffa de Panamá: woldai Lozada \& Freytag sp. nov., lamasi Lozada \& Freytag sp. nov. y grandis Lozada \& Freytag sp.nov. Además se dan a conocer siete nuevos registros para este país: variolaria Young, donsana Young, rubriguttata (Walker), elauta Young, dependens Young, uncata Young y trifasciata Cavichioli \& Chiamolera.

\section{Material y métodos}

Las especies nuevas descritas en este artículo fueron colectadas en varias localidades panameńas con trampas de luz colocadas para el trabajo científico del Dr. Henk Wolda del Smithsonian Tropical Research Institute (STRI). Los acrónimos de las instituciones donde es mantenido el material de Ladoffa reportado en este artículo es como sigue:

(STRI) Colección de Insectos, Smithsonian Tropical Research Institute, Balboa, Panamá.

(MIF) Museo de Invertebrados G.B. Fairchild, Universidad de Panamá.

(PMENT) Programa de Maestría en Entomología, Universidad de Panamá.

(MUSM)Departamento de Entomología, Museo de Historia Natural, Universidad Nacional Mayor de San Marcos, Lima, Perú.

La genitalia masculina para la determinación a nivel de especie fue preparada de la siguiente manera:

Separación del abdomen mediante la inserción de un estilete entre la junción del mismo y el tórax.
Inmersión del abdomen en $\mathrm{KOH}$ al $10 \%$ en caliente hasta lograr la despigmentación, el ablandamiento y el licuado del contenido interno.

Lavado en agua destilada para neutralizar la acción del álcali.

Colocación del abdomen así clarificado en microviales especiales para guardar genitalia en glicerina.

\section{Resultados}

\section{Ladoffa grandis Lozada \& Freytag, sp. nov.}

$$
\text { (Figs. 1 - 7) }
$$

Estructura.- Corona pronunciada anteriormente; fóvea media bien marcada, desde su base 2/3 la longitud media de la corona; ocelos sobre una línea imaginaria entre los ángulos oculares anteriores. Clípeo tumido, con su mitad anterior media aplanada, impresiones musculares claramente visibles; sutura entre clípeo y clipelo medialmente incompleta; en vista lateral, clipelo siguiendo el perfil del clípeo. Pronoto con márgenes laterales anteriormente convergentes, margen posterior rectilíneo.

Coloración.- Corona, pronoto y escutelo marrón claro. Corona con dos manchas rojizas no bien demarcadas, ápice y márgenes ántero-laterales amarillentos. Ocelos claros. Pronoto con tres bandas longitudinales rojizas, una central tocando el margen posterior pero no llegando al margen anterior, y dos laterales tocando ambos márgenes. Patrón de color del ala anterior como en $L$. dependens Young pero con la mancha claval intermedia en forma de "L". Cara amarillenta. Pleuras amarillas. Patas amarillas.

Genitalia masculina.- Pigófero bien producido, ápice redondeado, con macrosetas distribuidas en la mitad posterior; un proceso anterior dirigido mesalmente, adelgazado apicalmente, con una serie de denticulaciones en el extremo posterior. Placa subgenital triangular, casi llegando hasta el ápice del pigófero, borde externo con una hilera de macrosetas externamente. Estilo alargado, sobrepasando ampliamente el ápice del conectivo, el 

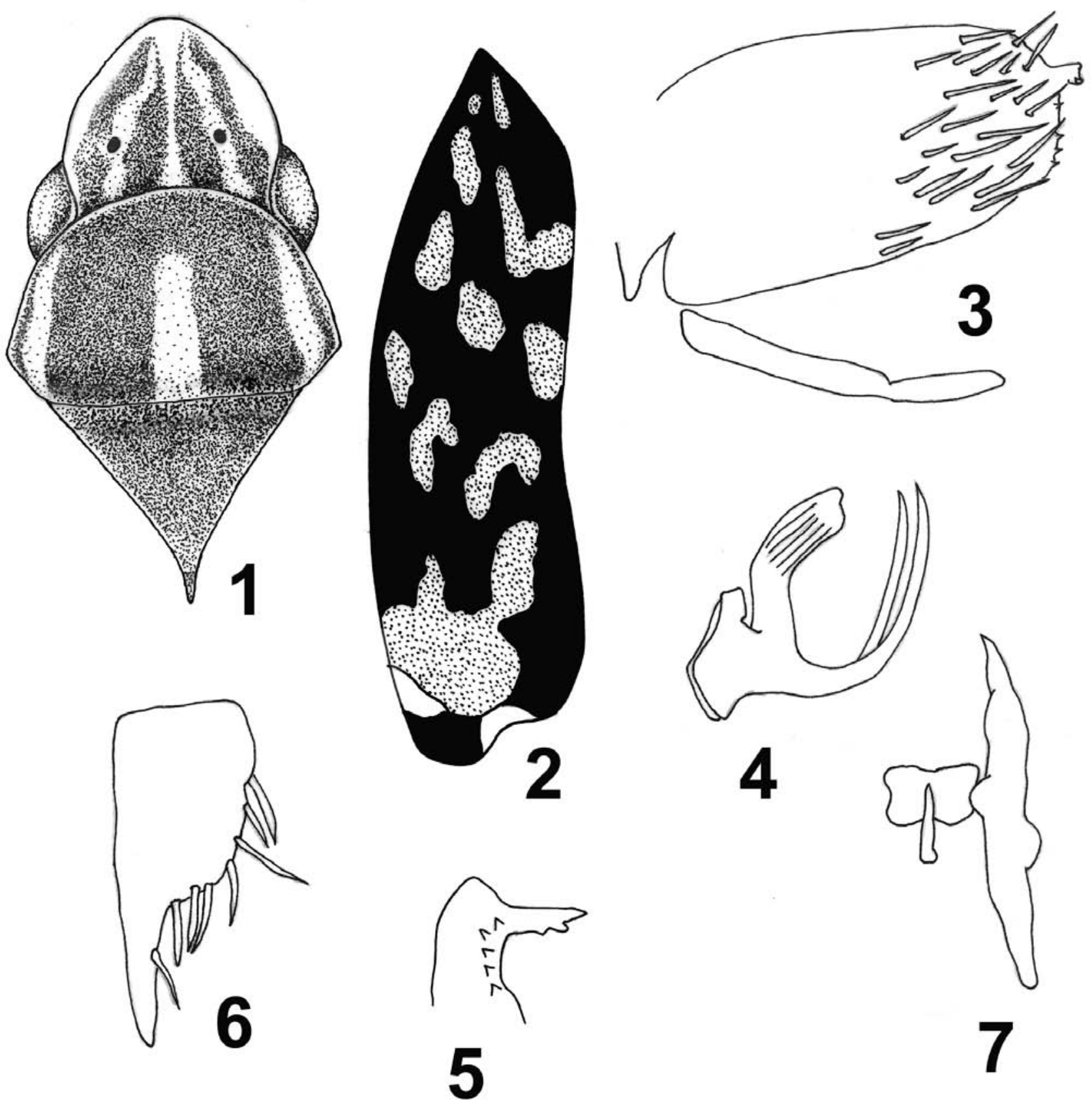

Figuras 1 - 7. Ladoffa grandis Lozada \& Freytag, sp. nov. (1) Dorso anterior, vista dorsal. (2) Ala anterior izquierda. (3) Pigófero y placa subgenital, vista lateral. (4) Edeago, vista lateral. (5) Proceso apical del pigófero, vista caudal. (6) Placa subgenital izquierda, vista ventral. (7) Estilo derecho y conectivo, vista dorsal.

cual es corto y en forma de "Y". Edeago como el de L. trifasciata Cavichioli \& Chiamolera, pero con los procesos basiventrales mucho más alargados.Paráfisis ausentes.

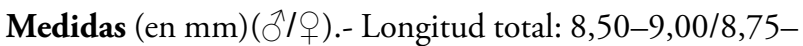
9,50; longitud media de la corona: $0,93-1,00 / 0,98-1,20$; longitud de la corona cercana a los ojos: $0,50-0,60 / 0,50-0,60$; longitud del pronoto: $1,20-1,27 / 1,25-1,30$; ancho del pronoto: 1,95-2,00/2,00-2,18; longitud del escutelo: 0,90-1,25/1,241,26; ancho basal del escutelo: 1,24-1,60/1,50-1,70; ancho interocular de la cabeza: $1,03-1,18 / 1,10-1,25$; ancho transocular de la cabeza: $1,70-1,76 / 1,79-1,80$; distancia entre los ocelos: $0,53-0,60 / 0,60-0,64$; distancia oceloocular: $0,26-0,30 / 0,30$.
Material Examinado.- Holotipo macho, Panamá, Chiriquí, Fortuna, $8^{\circ} 44^{\prime} \mathrm{N} 82^{\circ} 15^{\prime} \mathrm{W}, 23-27$. ix.1976, Henk Wolda coll.; 1 paratipo macho, mismos datos que el holotipo; 2 paratipos machos, mismos datos que el holotipo excepto 12-17 August 1976 y $20 . i v .1977$ y $1060 \mathrm{~m}$; 1 paratipo hembra, mismos datos que el holotipo; 3 paratipos hembras, mismos datos que el holotipo excepto 12-17 August 1976; 1 paratipo macho y 1 paratipo hembra, Trocha 3 de Noviembre, Bocas del Toro, iv.78, R.L. Dressler coll. Todos en STRI.

Comentarios.- Ladoffa grandis está relacionada a L. trifasciata Cavichioli \& Chiamolera por la configuración del edeago y la presencia de un proceso apical en el pigófero del macho. Difiere 

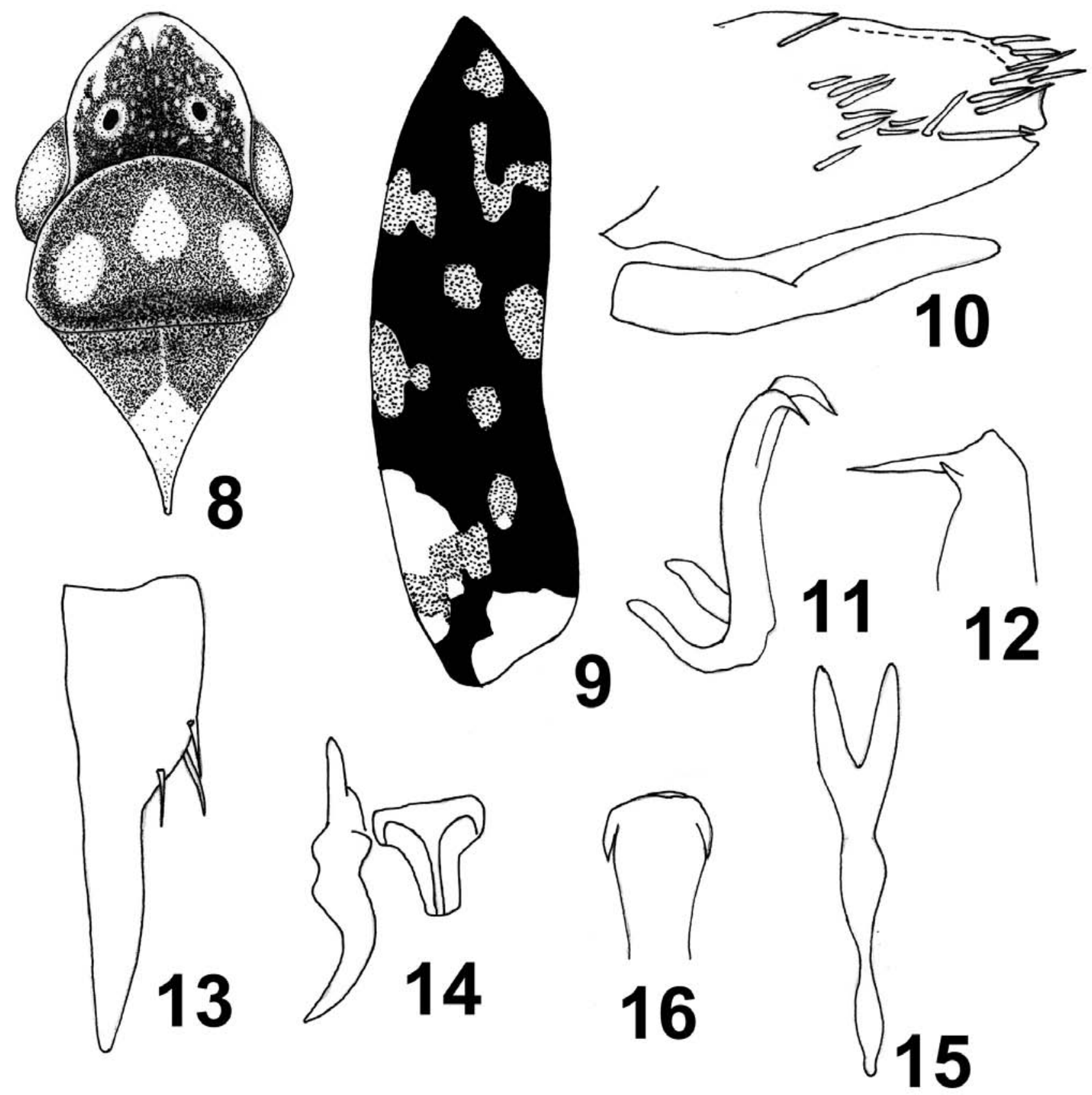

Figuras 8 - 16. Ladoffa woldai Lozada \& Freytag, sp. nov. (8) Dorso anterior, vista dorsal. (9) Ala anterior izquierda. (10) Pigófero y placa subgenital, vista lateral. (11) Edeago, vista látero-dorsal. (12) Proceso apical del pigófero, vista caudal. (13) Placa subgenital izquierda, vista ventral. (14) Estilo izquierdo y conectivo, vista dorsal. (15) Paráfisis, vista caudal. (16) Edeago, vista caudal.

porque los procesos basiventrales del edeago son más alargados y la diferente configuración de los procesos apicales del pigófero.

Etimología.- El nombre específico se refiere a que L. grandis es la especie de mayor tamaño del género.

\section{Ladoffa woldai Lozada \& Freytag, sp.nov.}

(Figs. $8-6$ )

Estructura.- Como en L. grandis.

Coloración.-Corona, pronoto y escutelo marrón oscuro, corona con pequeñas manchas circulares amarillentas distri- buidas en el disco y dos manchas pequeñas rojizas contiguas al margen de los ocelos; ápice y rebordes antenales amarillo pajizo. Pronoto con una mancha ovalada rojiza en la línea media y dos manchas oval-alargadas no tocando ambos márgenes. Escutelo con ápice amarillo pajizo, con un tinte rojizo. Patrón de color del ala anterior como en L. uncata Young. Cara, pleuras y patas amarillo marfil. Ocelos fuscos.

Genitalia masculina.- Pigófero bien desarrollado, con una hendidura apical más o menos profunda, macrosetas distribuidas en un grupo súperoapical y otro grupo sobre el disco; proceso dirigido mesalmente, puntiagudo, con una denticulación alargada en la base del proceso. Placa subgenital triangular, no 

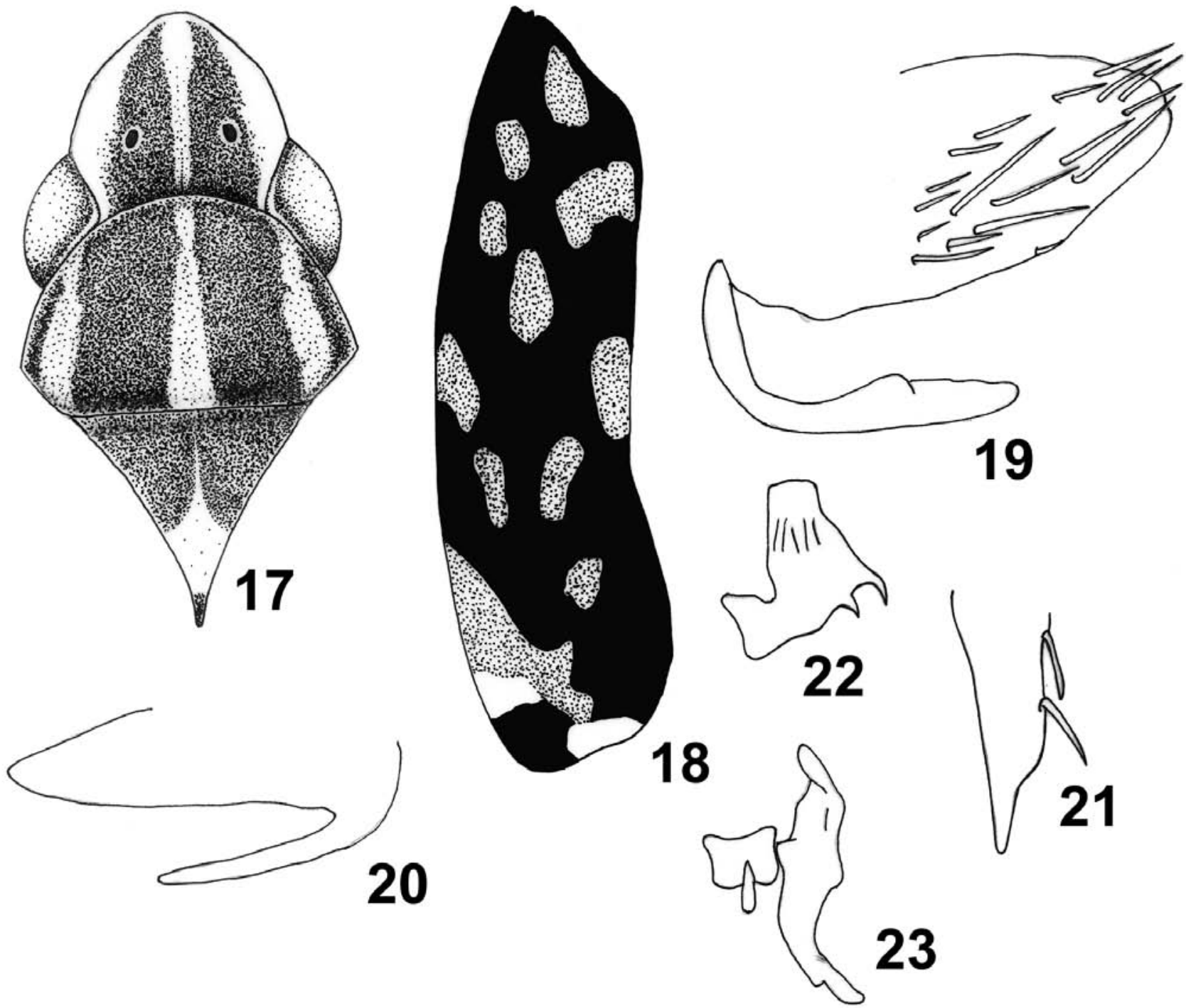

Figura 17 - 23. Ladoffa lamasi Lozada \& Freytag, sp. nov. (17) Dorso anterior, vista dorsal. (18) Ala anterior izquierda. (19) Pigófero y placa subgenital, vista lateral. (20) Pigófero y proceso basiventral, vista caudal. (21) Placa subgenital izquierda, vista ventral. (22) Edeago, vista lateral. (23) Estilo derecho y conectivo, vista dorsal.

sobrepasando el ápice del pigófero. Estilo sobrepasando el ápice del conectivo, el cual es corto y en forma de Y. Edeago con vaina arqueada centralmente, con dos procesos apicales puntiagudos, dirigidos apicalmente, ampliamente convergentes en vista caudal. Paráfisis presente, en forma de $\mathrm{Y}$, con la base bilobada.

Medidas (en mm) (ð) .- Longitud total: 7,00-7,30; longitud media de la corona: $0,90-0,94$; longitud de la corona cercana a los ojos: $0,38-0,42$; longitud del pronoto: $0.98-1,18$; ancho del pronoto: $1,63-1,70$; longitud del escutelo: $0,90-1,08$; ancho basal del escutelo: 1,26-1,40; ancho interocular de la cabeza: $0,90-1,00$; ancho transocular de la cabeza: 1,62-1,64; distancia entre los ocelos: 0,46-0,50; distancia oceloocular: 0,24-0,26.

Material examinado.- Holotipo macho, Panamá, Bocas del Toro, Corriente Grande, $100 \mathrm{~m}, 9^{\circ} 17^{\prime} 30^{\prime \prime} \mathrm{N}-82^{\circ} 32^{\prime} 41^{\prime \prime} \mathrm{W}$, 11.iii.1980, Henk Wolda coll.; 3 paratipos machos, mismos datos que el holotipo excepto 9.iv.1980, 21.ii.1980 y 24.ii.1980. Todos en STRI.

Comentarios.- Ladoffa woldai es relacionado a L. uncata Young por el patrón de color del ala anterior, la configuración de las paráfisis y por la presencia de procesos en el pigófero. Difiere por la diferente configuración del edeago.

Etimología.- La especie es dedicada al Dr. Henk Wolda por su sobresaliente trabajo de investigación en el Smithsonian Tropical Research Institute en Panamá.

\section{Ladoffa lamasi Lozada \& Freytag, sp.nov.}

(Figs. $17-23)$

Estructura.- Como en L. grandis.

Coloración.-Corona, pronoto y escutelo marrón oscuro, corona con una banda amarillo pajiza longitudinal media, rebordes antenales y márgenes ánterolaterales amarillo pajizos. Pronoto con tres bandas longitudinales amarillo-pajizas, una central y dos laterales, tocando ambos márgenes. Patrón de color del ala anterior como en L. rubriguttata (Walker). Cara, pleuras y patas amarillo-pajizas. Ocelos fuscos.

Genitalia masculina.- Pigófero bien desarrollado, margen 
posterior redondeado, con un proceso basiventral largo, no llegando al ápice del pigófero, macrosetas distribuidas en la mitad posterior. Placa subgenital triangular, bruscamente adelgazada apicalmente, no sobrepasando el ápice del pigófero. Estilo arqueado, sobrepasando el ápice del conectivo, el cual es corto y en forma de Y. Edeago como en L. rubriguttata pero con una espina ventral adicional. Paráfisis ausentes.

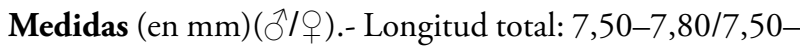
7,60; longitud media de la corona: $0,75-0,80 / 0,75-0,80$; longitud de la corona cercana a los ojos: 0,50/0,50; longitud del pronoto: 1,00/1,00; ancho del pronoto: $1,57-1,66 / 1,57-1,64$; longitud del escutelo: 0,86-1,00/0,86-1,00; ancho basal del escutelo: 1,18-1,20/1,18-1,25; ancho interocular de la cabeza: $0,85 / 0,85$; ancho transocular de la cabeza: $1,50 / 1,50$; distancia entre los ocelos: $0,40-0,50 / 0,40-0,50$; distancia oceloocular: $0,20 / 0,23-0,24$.

Material examinado.- Holotipo macho, Panamá, Bocas del Toro, Corriente Grande, $100 \mathrm{~m}, 9^{\circ} 17^{\prime} 30^{\prime \prime} \mathrm{N}-82^{\circ} 32^{\prime} 41^{\prime \prime W}$, 10.ii.1980, Henk Wolda coll.; 2 paratipos machos, mismos datos que el holotipo excepto 11 .iii.1980 y 7.ii.1980; 2 paratipos hembras, mismos datos que el holotipo excepto 16.xii.1979 y 3.iv.1980. Todos en STRI excepto el espécimen del 11.iii.1980 en MUSM.

Comentarios.- Ladoffa lamasi es relacionada a L. rubriguttata por la configuración del edeago, el patrón de color del ala anterior y la presencia de procesos en el pigófero. Difiere por la presencia de una espina ventral adicional en el edeago.

Etimología.- Esta especie es dedicada a la memoria del Ing. José Lamas Carrera, insigne entomólogo aplicado peruano.

\section{Nuevos registros de Ladoffa de Panamá}

1) Ladoffa variolaria Young:

Dos machos, Fortuna, 12-17.viii.76, H. Wolda coll. (STRI).

Dos hembras, Fortuna, Chiriquí, 12-17.viii.76, H. Wolda coll. (STRI).

Un macho, Cerro Colorado, Chiriquí, 24 km. NE San Félix, 1300 m., 12.vi.80, R. Pine coll. (STRI).

Un macho, Boquete, 1250 m., 2.viii.77, H. Wolda coll. (STRI).

Una hembra, Cerro El Calvario, 25-27.ii.79, J. Barría coll. (STRI).

Un macho, El Copé, 30.xi.80, light trap. (PMENT).

Un macho, El Copé, Coclé, 4.xi.80, E. Valdés coll. (MIF).

\section{2) Ladoffa donsana Young:}

Dos machos, El Copé, 2.xi y 4.xi.80, light trap. (PMENT). Dos machos, El Copé, Coclé, 2.xi.80, E. Valdés coll. (MIF).

Tres machos, Corriente Grande, 27.ii.80, 18.iv.80 y 9.iv.80, H. Wolda coll. (STRI).

\section{3) Ladoffa rubriguttata (Walker):}

Un macho, San Blas, near Punta Escocés, 1.iv.197831.i.1979, Caroline Ash coll. (STRI).

\section{4) Ladoffa elauta Young:}

Un macho y 1 hembra, Isla Colón, 14.i.76, H. Wolda coll. (STRI).

Un macho, Corriente Grande, 14.iv.80, H. Wolda coll. (STRI).

Una hembra, Corriente Grande, 14.i.80, H. Wolda coll. (STRI).

\section{5) Ladoffa dependens Young:}

Un macho, Corriente Grande, 14.ii.80, H. Wolda coll. (STRI).

\section{6) Ladoffa uncata Young:}

Una hembra, Pipeline Road, canopy knockdown, Luehea seemanni, 24.x.75 (STRI).

\section{7) Ladoffa trifasciata Cavichioli \& Chiamolera:}

Un macho y 2 hembras, Bocas del Toro, Isla Colón, 1.xi.78 y 14.i.76, H. Wolda coll. (STRI).

\section{Agradecimientos}

Al Dr. Henk Wolda por la invitación al primer autor para estudiar su colección de Cicadellidae mantenida en Pastor's House, Balboa, Panamá, y al Smithsonian Tropical Research Institute por la beca que permitió al autor principal una visita de trabajo de tres meses a Panamá.

\section{Literatura citada}

Basset Y. \& E. Charles. 2000. An annotated list of insect herbivores foraging on the seedlings of five forest trees in Guyana. An. Soc. Entomol. Bras. 29: 433-452.

Cavichioli R.R. \& L. de Bortolli Chiamolera. 2001. Trés espécies novas de Ladoffa Young (Hemiptera, Auchenorrhyncha, Cicadellidae). Rev. Bras. Zool. 18(1): 285-291.

Lozada P.W. 1991 (1993). Una nueva especie de Ladoffa Young, 1977 (Homoptera: Cicadellidae). Rev. Per. Ent. 34: 61-62.

Young D.A. 1977. Taxonomic study of the Cicadellinae (Homoptera: Cicadellidae). Part 2. New World Cicadellini and the genus Cicadella. N. Carol. Agric. Exp. Stat. Bull. 239: 1-1135. 


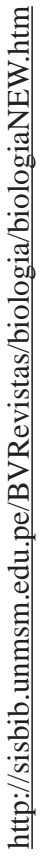

